\title{
Ácaros associados ao cafeeiro (Coffea spp.) no estado de São Paulo, Brasil. Parte I. Mesostigmata
}

\author{
Jeferson Luiz de Carvalho Mineiro ${ }^{1,3}$, Adalton Raga ${ }^{1}$, Mario Eidi Sato ${ }^{1}$ \& Antonio Carlos Lofego ${ }^{2}$ \\ ${ }^{1}$ Laboratório Entomologia Econômica, Instituto Biológico - CEIB, \\ Rod. Heitor Penteado, Km 3, CP 70, CEP 13001-970, Campinas, SP, Brasil \\ ${ }^{2}$ Departamento de Zoologia e Botânica, Universidade Estadual Paulista - UNESP, \\ Rua Cristóvão Colombo, 2265, CEP 15054-000, São José do Rio Preto, SP, Brasil \\ ${ }^{3}$ Autor para correspondência: Jeferson Luiz,de Carvalho Mineiro,e-mail: jefmin@ hotmail.com
}

\begin{abstract}
MINEIRO, J.L.C., RAGA, A., SATO, M.E. \& LOFEGO, A.C. 2009. Mites associated with coffee plants (Coffea spp.) in the state of São Paulo, Brazil. Part I. Mesostigmata. Biota Neotrop. 9(1): http://www.biotaneotropica. org.br/v9n1/en/abstract?article+bn00709012009.
\end{abstract}

\begin{abstract}
The objective of this study was to carry out a survey of mite species belonging to the order Mesostigmata on Coffea spp. in different localities of the State of São Paulo. Samples of coffee (Coffea spp.) leaves and branches were collected in 27 municipalities of the State of São Paulo, in different periods of the year. All collected mites were mounted in Hoyer's medium on microscope slides. The identification of mites was at species level when possible. Thirty-nine mite species were identified, belonging to the families Ameroseiidae, Ascidae, Laelapidae, Macrochelidae, Parasitidae, Phytoseiidae and to a group for which the family is still not defined, Africoseius sp. Keywords: Acari, coffee, taxonomy.
\end{abstract}

MINEIRO, J.L.C., RAGA, A., SATO, M.E. \& LOFEGO, A.C. 2009. Ácaros associados ao cafeeiro (Coffea spp.) no estado de São Paulo, Brasil. Parte I. Mesostigmata. Biota Neotrop. 9(1): http://www.biotaneotropica. org.br/v9n1/pt/abstract?article+bn00709012009.

Resumo: Este trabalho teve como objetivo fazer um levantamento das espécies de ácaros pertencentes à ordem Mesostigmata em Coffea spp. em diferentes localidades do estado de São Paulo. Folhas e ramos de cafeeiros (Coffea spp.) foram coletados em 27 municípios do estado de São Paulo e em diferentes épocas do ano. Todos os ácaros encontrados foram montados em lâminas de microscopia, em meio de Hoyer. A identificação dos ácaros foi feita até o nível específico quando possível. Foram identificadas 39 espécies de ácaros pertencentes às famílias Ameroseiidae, Ascidae, Laelapidae, Macrochelidae, Parasitidae, Phytoseiidae e a um grupo que ainda está sem uma família definida, Africoseius sp.

Palavras-chave: Acari, café, taxonomia. 


\section{Introdução}

Em contraste com a diversidade de famílias de ácaros predadores da ordem Prostigmata, os ácaros predadores mais comumente encontrados sobre plantas pertencem aos Phytoseiidae (ordem Mesostigmata), família constituída de várias espécies que podem ser usadas como eficientes agentes de controle biológico de grupos importantes de ácaros fitófagos de Tetranychidae, Tenuipalpidae, Tarsonemidae e Eriophyoidea (Gerson et al. 2003). Ácaros Mesostigmata da família Ascidae também apresentam grande diversidade de espécies, sendo suplantados apenas pelos Phytoseiidae. A ocorrência da família sobre plantas é observada principalmente em regiões úmidas do globo terrestre, sendo Lasioseius spp. e Asca spp. os grupos mais freqüentemente encontrados sobre folhas (Moraes \& Flechtmann 2008). Espécies destes dois gêneros são comumente encontradas associadas a Eriophyioidea e Tenuipalpidae, podendo atuar no controle natural destes ácaros (Walter et al. 1993), mas estudos visando a utilização de Ascidae no controle de pragas têm sido relativamente poucos (Moraes \& Flechtmann 2008).

A diversidade de ácaros que ocorrem em cafeeiros no Brasil ainda é pouco conhecida, porém em trabalhos mais recentes tem evidenciado que é alta, não somente para predadores quanto para pragas e de outros hábitos alimentares pouco conhecidos (Spongoski et al. 2005, Mineiro et al. 2001 e 2006). Algumas espécies de ácaros predadores das famílias Phytoseiidae, Ascidae, Bdellidae, Cunaxidae, Cheyletidae, Raphignathidae e Stigmaeidae são conhecidas em Coffea spp. (Flechtmann 1967b e 1968, Pallini Filho et al. 1992, Mineiro et al. 2001 e 2006, Spongoski et al. 2005).

Este trabalho teve como objetivo realizar um levantamento das espécies de ácaros pertencentes à ordem Mesostigmata em Coffea spp., em diferentes localidades do Estado de São Paulo.

\section{Material e Métodos}

O levantamento foi realizado em 27 municípios do Estado de São Paulo, entre 2001 e 2007 (Tabela 1). Neste trabalho também foram incluídas as espécies já relatadas para os municípios de Garça e Jeriquara (Mineiro et al. 2006). O material (folhas e ramos) foi coletado em plantios comerciais e não comercias. As folhas de cafeeiro (Coffea spp.) foram coletadas, acondicionadas em sacos plásticos e transportadas em caixas de poliestireno para o Laboratório de Entomologia Econômica do Instituto Biológico em Campinas, SP (LEE-IB). Para a remoção dos ácaros, as folhas foram mergulhadas em recipientes plásticos contendo uma solução de álcool (70\%), onde permaneceram imersas por 10 minutos (Spongoski et al. 2005, Mineiro et al. 2006). Decorrido este prazo, as folhas foram agitadas na solução para que os ácaros se desprendessem e se mantivessem no líquido do recipiente. Em seguida, essa solução foi passada em peneira (malha de $0,038 \mathrm{~mm}$ ) e o material retido foi transferido para frascos de vidro com álcool $70 \%$ para posterior triagem. Todos os ácaros encontrados foram montados em lâminas de microscopia com meio de Hoyer. A identificação dos ácaros foi feita até o nível específico quando possível.

Para os fitoseídeos, utilizou-se o sistema de classificação genérico proposto por Chant \& Yoshida-Shaul (1983), Chant \& McMurtry (1994) e Moraes et al. (2004) e para os representantes da família Ascidae foi utilizado Lindquist \& Evans (1965) e Halliday et al. (1998).

Os números romanos após cada data de coleta indicam o número de exemplares examinados e as letras $\mathrm{f}, \mathrm{m}$, pn, dn significam: fêmea, macho, protoninfa e deutoninfa, respectivamente. Uma amostra representativa dos espécimes encontrados está depositada na coleção de referência de ácaros Geraldo Calcagnolo do LEE-IB.
Tabela 1. Coordenadas geográficas e altitudes dos municípios no estado de São Paulo onde foram realizadas as coletas.

Table 1. Geographic coordinates and altitudes of the municipalities in the State of São Paulo where the mites were collected.

\begin{tabular}{|c|c|c|}
\hline Município & Coordenadas & $\begin{array}{c}\text { Altitude } \\
\text { (m) }\end{array}$ \\
\hline Assis & $22^{\circ} 39^{\prime} 39^{\prime \prime} \mathrm{S}$ e $50^{\circ} 25^{\prime} 13^{\prime \prime} \mathrm{W}$ & 560 \\
\hline Atibaia & $23^{\circ} 06^{\prime} 59^{\prime \prime} \mathrm{S}$ e $46^{\circ} 33^{\prime} 06^{\prime \prime} \mathrm{W}$ & 800 \\
\hline Campinas & $22^{\circ} 53^{\prime} 20^{\prime \prime} \mathrm{S}$ e $47^{\circ} 04^{\prime} 40^{\prime \prime} \mathrm{W}$ & 680 \\
\hline Cândido Mota & $22^{\circ} 44^{\prime} 52^{\prime \prime} \mathrm{S}$ e $50^{\circ} 23^{\prime} 08^{\prime \prime} \mathrm{W}$ & 460 \\
\hline Cristais Paulista & $20^{\circ} 24^{\prime} 22^{\prime \prime} \mathrm{S}$ e $47^{\circ} 25^{\prime} 13^{\prime \prime} \mathrm{W}$ & 980 \\
\hline Dois Córregos & $22^{\circ} 21^{\prime} 53^{\prime \prime} \mathrm{S}$ e $48^{\circ} 22^{\prime} 50^{\prime \prime} \mathrm{W}$ & 677 \\
\hline Franca & $20^{\circ} 32^{\prime} 18^{\prime \prime} \mathrm{S}$ e $47^{\circ} 24^{\prime} 06^{\prime \prime} \mathrm{W}$ & 1000 \\
\hline Gabriel Monteiro & $21^{\circ} 31^{\prime} 51^{\prime \prime} \mathrm{S}$ e $50^{\circ} 33^{\prime} 24^{\prime \prime} \mathrm{W}$ & 420 \\
\hline Garça & $22^{\circ} 12^{\prime} 55^{\prime \prime} \mathrm{S}$ e $49^{\circ} 39^{\prime} 04^{\prime \prime} \mathrm{W}$ & 682 \\
\hline Indaiatuba & $23^{\circ} 05^{\prime} 12^{\prime \prime} \mathrm{S}$ e $47^{\circ} 13^{\prime} 06^{\prime \prime} \mathrm{W}$ & 640 \\
\hline Itirapuã & $20^{\circ} 38^{\prime} 30^{\prime \prime} \mathrm{S}$ e $47^{\circ} 13^{\prime} 09^{\prime \prime} \mathrm{W}$ & 860 \\
\hline Itupeva & $23^{\circ} 09^{\prime} 12^{\prime \prime} \mathrm{S}$ e $47^{\circ} 03^{\prime} 28^{\prime \prime} \mathrm{W}$ & 675 \\
\hline Jaboticabal & $21^{\circ} 15^{\prime} 20^{\prime \prime} \mathrm{S}$ e $48^{\circ} 19^{\prime} 16^{\prime \prime} \mathrm{W}$ & 583 \\
\hline Jaguariúna & $22^{\circ} 42^{\prime} 18^{\prime \prime} \mathrm{S}$ e $46^{\circ} 59^{\prime} 22^{\prime \prime} \mathrm{W}$ & 580 \\
\hline Jeriquara & $20^{\circ} 18^{\prime} 42^{\prime \prime} \mathrm{S} \mathrm{e} 47^{\circ} 35^{\prime} 20^{\prime \prime} \mathrm{W}$ & 860 \\
\hline Lutécia & $22^{\circ} 20^{\prime} 24^{\prime \prime} \mathrm{S}$ e $50^{\circ} 23^{\prime} 32^{\prime \prime} \mathrm{W}$ & 580 \\
\hline Maracaí & $22^{\circ} 36^{\prime} 39^{\prime \prime} \mathrm{S}$ e $50^{\circ} 40^{\prime} 01^{\prime \prime} \mathrm{W}$ & 380 \\
\hline Mococa & $21^{\circ} 28^{\prime} 16^{\prime \prime} \mathrm{S}$ e $47^{\circ} 00^{\prime} 23^{\prime \prime} \mathrm{W}$ & 620 \\
\hline Monte Alegre do Sul & $22^{\circ} 40^{\prime} 50^{\prime \prime} \mathrm{S}$ e $46^{\circ} 40^{\prime} 45^{\prime \prime} \mathrm{W}$ & 760 \\
\hline Monte Mor & $22^{\circ} 56^{\prime} 47^{\prime \prime} \mathrm{S}$ e $47^{\circ} 18^{\prime} 58^{\prime \prime} \mathrm{W}$ & 560 \\
\hline Olímpia & $20^{\circ} 44^{\prime} 16^{\prime \prime} \mathrm{S}$ e $48^{\circ} 55^{\prime} 02^{\prime \prime} \mathrm{W}$ & 500 \\
\hline Paranapuã & $20^{\circ} 06^{\prime} 15^{\prime \prime} \mathrm{S}$ e $50^{\circ} 35^{\prime} 12^{\prime \prime} \mathrm{W}$ & 600 \\
\hline Pindamonhangaba & $22^{\circ} 55^{\prime} 33^{\prime \prime} \mathrm{S}$ e $45^{\circ} 27^{\prime} 40^{\prime \prime} \mathrm{W}$ & 540 \\
\hline Piracicaba & $22^{\circ} 42^{\prime} 30^{\prime \prime} \mathrm{S}$ e $47^{\circ} 38^{\prime} 01^{\prime \prime} \mathrm{W}$ & 554 \\
\hline São Paulo & $23^{\circ} 32^{\prime} 52^{\prime \prime} \mathrm{S}$ e $46^{\circ} 38^{\prime} 07^{\prime \prime} \mathrm{W}$ & 860 \\
\hline Tatuí & $23^{\circ} 21^{\prime} 03^{\prime \prime} \mathrm{S}$ e $47^{\circ} 50^{\prime} 53^{\prime \prime} \mathrm{W}$ & 620 \\
\hline Tietê & $23^{\circ} 06^{\prime} 03^{\prime \prime} \mathrm{S}$ e $47^{\circ} 42^{\prime} 55^{\prime \prime} \mathrm{W}$ & 500 \\
\hline
\end{tabular}

\section{Resultados}

Foram identificadas várias espécies de Mesostigmata pertencentes às famílias Ameroseiidae, Ascidae, Laelapidae, Macrochelidae, Parasitidae, Phytoseiidae e um grupo que ainda está sem uma família definida, Africoseius sp.

São apresentadas, a seguir, as famílias, gêneros e espécies de ácaros encontrados nas várias espécies e cultivares de Coffea spp. coletadas em diferentes localidades no Estado de São Paulo.

\section{AMEROSEIIDAE Hughes, 1961}

\section{Ameroseius sp.}

Espécime examinado: Franca, Fazenda da Mata, C. arabica cv. Catuaí Vermelho, 20.ii.2003, 1f.

\section{Kleemannia sp.}

Espécimes examinados: Jeriquara, Fazenda São Francisco, C. arabica cv. Catuaí Amarelo, 19.ii.2003, 5m, 22f; 15.v.2003, 2f; 20.vi.2003, 1 f. 
ASCIDAE Voigts \& Oudemans, 1905

\section{Aceodromus convolvuli Muma, 1961}

Aceodromus convolvuli Muma, 1961: 282, Lindquist \& Chant, 1964: 502.

Espécimes examinados: Jeriquara, Fazenda Boa Esperança, C. arabica cv. Mundo Novo, 16.ii.2002, 1f; 23.xi.2002, 1f; 21.xii.2002, 1f; 18.i.2003, 2f; 31.i.2003, 1f; 16.ii.2003, 1f.

Relatos prévios no Brasil: Bahia, Ceará e Piauí (Moraes et al. 1993; Rodrigues et al. 1996).

2. Asca sp.

Espécimes examinados: Jeriquara, Fazenda Boa Esperança, C. arabica cv. Mundo Novo, 26.iv.2003, 2f, 2pn; 21.v.2003, 1f, 1m; Fazenda São Francisco, C. arabica cv. Catuaí Amarelo, 20.iii.2003, 5f; 17.i.2003, 2f; Franca, Fazenda da Mata, C. arabica cv. Catuaí Vermelho, 20.ii.2003, 2f.

3. Blattisocius tarsalis (Berlese, 1918)

Lasioseius tarsalis Berlese, 1918: 134.

Seiulus muricatus sensu Oudemans not Koch. - Burkhardt, 1920:54.

Sejus muricatus sensu Oudemans not Koch. - Zacher, 1927: 254.

Typhlodromus tineivorus Oudemans, 1929: 34. Sinonímia de acordo com Evans (1958).

Blattisocius triodons Keegan, 1944: 181. - Garman, 1948: 18, Nesbitt, 1951:51. Sinonímia de acordo com Baker \& Warton (1952).

Lasioseius similis Schweizer, 1949: 49. Sinonímia de acordo com Evans (1958).

Blattisocius tineivorus - Nesbitt, 1951: 51, Womersley, 1954: 184.

Melichares (Blattisocius) tarsalis - Evans, 1958: 209, Athias-Henriot, 1959: 162, Hughes, 1961: 235, Champ, 1965: 236, Champ, 1966: 206.

Blattisocius tarsalis - Schweizer, 1961: 132, Chant, 1963: 298, Westerboer \& Bernhard, 1963: 745, Lindquist \& Evans, 1965: 48, McGraw \& Farrier, 1969: 59, Treat, 1975: 96, Hughes, 1976: 325, Domrow, 1979: 98, Haines, 1979: 20, Flechtmann, 1986: 69, Karg, 1993: 172, Halliday et al. 1998:19.

Espécimes examinados: Jeriquara, Fazenda Boa Esperança, C. arabica cv. Mundo Novo, 23.xi.2002, 1 f.

Relatos prévios no Brasil: Pernambuco, São Paulo (Reis \& Paschoal 1968, Flechtmann 1981, Mineiro \& Moraes 2001, Souza et al. 2005).

\section{Blatttisocius sp.}

Espécimes examinados: Jeriquara, Fazenda Boa Esperança, C. arabica cv. Mundo Novo, 06.ix.2001, 1m; Garça, Estação Experimental Alcides Carvalho, C. arabica cv. Mundo Novo, 10.i.2003, 1m.

5. Gamasellodes sp.

Espécimes examinados: Jeriquara, Fazenda Boa Esperança, C. arabica cv. Mundo Novo, 17.xi.2001, 1dn; 16.ii.2002, 1dn; 11.v.2002, 2dn; 07.i.2003, 14dn e 18.i.2003, 1dn; Garça, Estação Experimental Alcides Carvalho, C. arabica cv. Icatu Amarelo, 04.v.2002, $1 \mathrm{dn}$.

6. Lasioseius sp.

Espécimes examinados: Jeriquara, Fazenda Boa Esperança, C. arabica cv. Mundo Novo, 04.i.2001, 1m e 14.x.2002, 1f; Garça, Estação Experimental Alcides Carvalho, C. canephora cv.
Apoatã, 07.viii.2001, 1f; C. arabica cv. Mundo Novo, 13.ii.2003, 1f; Monte Alegre do Sul, Sítio São José, C. arabica cv. Mundo Novo, 09.iii.2005, 1f.

7. Leioseius sp.

Espécime examinado: Jeriquara, Fazenda Boa Esperança,

C. arabica cv. Mundo Novo, 25.v.2002, $1 \mathrm{dn}$.

8. Proctolaelaps bickleyi (Bram, 1956)

Garmania bickleyi Bram, 1956: 292.

Proctolaelaps striata Westerboer, 1963: 356. Sinonímia de acordo com Lindquist \& Hunter (1965).

Garmania domestica Nesbitt, 1951: 44.

Proctolaelaps bickleyi Chant, 1963: 269, Lindquist \& Hunter, 1965:16, McGraw \& Farrier, 1969: 72, Karg, 1985: 192, Karg, 1988: 448, Halliday et al., 1998: 31.

Espécimes examinados: Garça, Estação Experimental Alcides Carvalho, C. arabica cv. Catuaí Amarelo, 10.x.2001, 1f; Monte Alegre do Sul, Sítio São José, C. arabica cv. Mundo Novo, 05.v.2004, 1f.

Relatos prévios no Brasil: São Paulo (Flechtmann 1976).

9. Proctolaelaps $\mathrm{sp} .1$

Espécimes examinados: Jeriquara, Fazenda Boa Esperança, C. arabica cv. Mundo Novo, 11.xi.2002, $1 \mathrm{~m}$ e 15.iii.2003, $1 \mathrm{f}$.

\section{Proctolaelaps sp. 2}

Espécime examinado: Jeriquara, Fazenda Boa Esperança, C. arabica cv. Mundo Novo, 19.vii.2002, 1f.

\section{LAELAPIDAE Berlese, 1892}

\section{Androlaelaps sp.}

Espécimes examinados: Jeriquara, Fazenda São Francisco, C. arabica cv. Catuaí Amarelo, 11.i.2003, 1f; Garça, Estação Experimental Alcides Carvalho, C. arabica cv. Mundo Novo, 01.iv.2002, 1f.

\section{Pseudoparasitus sp.}

Espécime examinado: Jeriquara, Fazenda Boa Esperança, C. arabica cv. Mundo Novo, 07.xii.2002, 1f.

\section{MACROCHELIDAE Vitzthum, 1930}

\section{Macrocheles sp.}

Espécimes examinados: Jeriquara, Fazenda Boa Esperança, C. arabica cv. Mundo Novo, 12.iv.2002, 1f; Garça, Estação Experimental Alcides Carvalho, C. arabica cv. Mundo Novo, 05.ii.2002, 1f e Icatu Amarelo, 03.x.2002, 1f.

PARASITIDAE Oudemans, 1901

Espécimes examinados: Jeriquara, Fazenda Boa Esperança, C. arabica cv. Mundo Novo, 01.xii.2001, 1dn; 04.ii.2002, 1dn; 07.xii.2002, 1dn; Fazenda Boa Esperança, C. arabica cv. Catuaí Amarelo, 17.i.2003, 1dn.

PHYTOSEIIDAE Berlese, 1913

\section{Amblyseiella setosa Muma, 1955}

Amblyseiella setosa Muma, 1955: 266, Muma, 1961: 286, Muma \& Denmark, 1970: 54, Moraes et al., 2004: 9.

Phytoseiullus setosa (Muma) Garman, 1958: 71.

Typhlodromus (Amblyseius) setosus (Muma) Chant, 1959: 70. Espécime examinado: Jeriquara, Fazenda Boa Esperança, C. arabica cv. Mundo Novo, 26.x.2002, 1f.

Relatos prévios no Brasil: São Paulo (Mineiro et al. 2005). 


\section{Amblyseius aerialis (Muma, 1955)}

Amblyseiopsis aerialis Muma, 1955: 264, Garman, 1958: 75 Typhlodromus (Amblyseius) aerialis (Muma) Chant, 1959: 88. Amblyseius aerialis, Athias-Henriot, 1957: 338, Muma, 1961: 287, De Leon, 1966: 91, Muma \& Denmark, 1970: 66, Denmark \& Muma, 1973: 249, Moraes et al., 1986:15, Moraes \& Mesa, 1988: 71, Denmark \& Muma, 1989, 15, Kreiter \& Moraes, 1997: 377, Feres \& Moraes, 1998: 126, Moraes et al., 1999: 238, Gondim Jr. \& Moraes, 2001: 67, Moraes et al., 2004: 13, Feres et al., 2005: 3, Vasconcelos et al., 2006: 92, Buosi et al., 2006: 3.

Espécimes examinados: Jeriquara, Fazenda Boa Esperança, C. arabica cv. Mundo Novo, 12.iv.2002, 1f, 11.v.2002, 1f e 01.xii.2001, 1f; Fazenda São Francisco, C. arabica cv. Mundo Novo, 15.ii.2002, 2f, 06.iv.2002, 1f, cv. Catuai Amarelo, 18.xii.2002, 1f e 17.iv.2003, 2f; Garça, Estação Experimental Alcides Carvalho, C. arabica cv. Catuai Amarelo, 09.viii.2002, 1f.

Relatos prévios no Brasil: Bahia, Pernambuco e São Paulo (Denmark \& Muma 1973, Moraes et al. 1993, Moraes \& Oliveira 1982, Feres \& Moraes 1998, Barbosa et al. 2003, Vasconcelos et al. 2006, Buosi et al. 2006).

\section{Amblyseius chiapensis De Leon, 1961}

Amblyseius chiapensis De Leon, 1961: 85, De Leon, 1962: 175, McMurtry, 1983: 250, Moraes \& Mesa, 1988: 72, Denmark \& Muma, 1989: 94, McMurtry \& Moraes, 1989: 185, Gondim Jr. \& Moraes, 2001: 67, Ferla \& Moraes, 2002: 1013, Moraes et al., 2004: 19, Lofego et al., 2004: 3, Feres et al., 2005: 3, Buosi et al., 2006: 3.

Amblyseius triplaris De Leon, 1967: 25 (Sinonímia de acordo com Denmark \& Muma, 1989: 94).

Espécimes examinados: Itupeva, C. arabica, 26.ii.2004, 2f; Maracaí, Sítio Santa Margarida, C. arabica, 31.v.2006, 1f; Cristais Paulista, C. arabica, 21.i.2004, 1 f.

Relatos prévios no Brasil: Bahia, Ceará, Maranhão, Pernambuco, Rio Grande do Sul, Santa Catarina e São Paulo (Sato et al. 1994, Moraes et al. 1993, Rodrigues et al. 1996, Santana \& Flechtmann 1998, Ferla \& Moraes 1998 e 2002, Lorenzato 1987 e 1988, Fenilli \& Flechtmann 1990, Denmark \& Muma 1973, Gondim Jr. \& Moraes 2001, Lofego et al. 2004, Feres et al. 2005, Buosi et al. 2006).

\section{Amblyseius compositus Denmark \& Muma, 1973}

Amblyseius compositus Denmark \& Muma, 1973: 240, 1989: 95, Moraes et al., 1986: 11, Gondim Jr. \& Moraes, 2001: 67, Moraes et al., 2004: 21, Feres et al., 2005: 3, Vasconcelos et al., 2006: 92.

Espécimes examinados: Atibaia, Sítio Jorge Kurosawa, C. arabica cv. Catuaí Amarelo, 13.vi.2000, 1f; Piracicaba, ESALQ/USP, C. arabica, 12.viii.2003, 1f; Monte Alegre do Sul, Pólo Regional do Leste Paulista, C. arabica sob mata, 01.xii.2006, 1f.

Relatos prévios no Brasil: Bahia, Minas Gerais, Pernambuco, Piauí (Moraes et al. 1993, Rodrigues et al. 1996, Reis et al. 2000, Mineiro et al. 2004, Lofego et al. 2004: 3, Vasconcelos et al. 2006).

\section{Amblyseius curiosus (Chant \& Baker, 1965)}

Iphiseius curiosus Chant \& Baker, 1965: 11.

Amblyseius curiosus Muma \& Denmark, 1970: 64, Denmark \& Muma, 1989: 35, Moraes et al., 2004: 21.

Amblyseius arenus Muma, 1965: 250 (sinonímia de acordo com Muma et al., 1970).
Espécimes examinados: Jeriquara, Fazenda Boa Esperança, C. arabica cv. Mundo Novo, 04.iv.2001, 1f e 18.iv.2001, 2f.

Relatos prévios no Brasil: São Paulo (Flechtmann 1967a, Mineiro \& Moraes 2001).

6. Amblyseius herbicolus (Chant, 1959)

Typhlodromus (Amblyseius) herbicolus Chant, 1959: 84.

Amblyseius herbicolus; Daneshvar \& Denmark, 1982: 5, McMurtry \& Moraes, 1984: 34, Denmark \& Muma, 1989: 59, Zacarias \& Moraes, 2001: 580, Ferla \& Moraes, 2002: 1013, Moraes et al., 2004: 27, Vasconcelos et al., 2006: 92.

Amblyseius impactus Chaudhri, 1968: 553 (Sinonímia de acordo com Daneshvar \& Denmark, 1982: 5).

Amblyseius deleoni Muma \& Denmark, 1970: 68 in Muma et al., 1970: 68 (Sinonímia de acordo com Daneshvar \& Denmark, 1982: 5).

Espécimes examinados: Atibaia, Sítio Kurosawa, C. arabica cv. Catuaí Amarelo, 13.vi.2000, 7f; 27.vi.2000, 2f; 11.vii.2000, 1f e 25.vii.2000, 9f; Garça, Estação Experimental Alcides Carvalho, C. canephora cv. Apoatã, 09.x.2001, 1f; Jeriquara, Fazenda Boa Esperança, C. arabica cv. Mundo, 02.iii.2002, 2f; Fazenda São Francisco, C. arabica cv. Mundo Novo, 23.ii.2002, If e 08.iii.2002, 2f; C. arabica cv. Catuaí Amarelo, 20.iii.2003, If e 17.iv.2003, 3f; Franca, Fazenda da Mata, C. arabica cv. Catuaí Vermelho, 20.ii.2003, 6f; São Paulo, Instituto Biológico, C. arabica, 05.xi.2003, 2f; Monte Alegre do Sul, Sítio São José, C. arabica cv. Mundo Novo, 23.xii.2004, 1f; Monte Alegre do Sul, Pólo Regional do Leste Paulista, C. arabica, 20.vi.2006, $10 f$.

Relatos prévios no Brasil: Minas Gerais, Pernambuco, São Paulo, Rio Grande do Sul (Pallini Filho et al. 1992, Reis et al. 2000, Mineiro \& Moraes 2001, Ferla \& Moraes 2002, Zacarias \& Moraes 2002, Mineiro \& Raga 2003, Mineiro et al. 2004, Spongoski et al. 2005, Vasconcelos et al. 2006).

\section{Euseius alatus De Leon, 1966}

Euseius alatus De Leon, 1966: 87, Denmark \& Muma, 1973: 262, Moraes \& McMurtry, 1983: 137, Feres \& Moraes, 1998: 127, Zacarias \& Moraes, 2001: 581, Ferla \& Moraes, 2002: 1015, Moraes et al., 2004: 60, Furtado et al., 2005: 134, Vasconcelos et al., 2006: 93.

Euseius paraguayensis Denmark \& Muma, 1970: 224 (Sinonímia, de acordo com Moraes \& McMurtry, 1983: 137).

Espécimes examinados: Campinas, Instituto Agronômico de Campinas, C. Canephora cv. Guarini, 02.ix.2003, 4f, Coffea liberica var. Piatã, 02.ix.2003, 2f; Monte Alegre do Sul, Sítio São José, C. arabica cv. Mundo Novo, 12.xi.2004, 4f; 23.xii.2004, 2f; Monte Alegre do Sul, Polo Regional do Leste Paulista, C. arabica do interior de mata, 20.vi.2006, 5f; Socorro, Sítio São Miguel, C. arabica, 25.v.2006, 1f; Monte Alegre do Sul, Sítio São José, C. arabica cv. Mundo Novo, 05.v.ii.2005, 1f.

Relatos prévios no Brasil: Bahia, Ceará, Maranhão, Minas Gerais, Pernambuco, Rio Grande do Sul, São Paulo (Moraes et al. 1993, Moraes \& McMurtry 1983, Pallini Filho et al. 1992, Sato et al. 1994, Rodrigues et al. 1996, Reis et al. 2000, Zacarias \& Moraes 2002, Ferla \& Moraes 2002, Barbosa et al. 2003, Furtado et al. 2005, Vasconcelos et al. 2006).

8. Euseius citrifolius Denmark \& Muma, 1970

Euseius citrifolius Denmark \& Muma, 1970: 222, Moraes \& McMurtry, 1983: 138, Moraes et al., 1991: 131, Feres \& Moraes, 1998: 127, Zacarias \& Moraes, 2001: 581, Ferla \& Moraes, 2002 : 1016, Moraes et al., 2004: Lofego et al., 2004: 4, Bellini et al., 
2005: 37, Demite \& Feres, 2005: 831, Furtado et al., 2005: 134, Buosi et al., 2006: 4.

Espécimes examinados: Jeriquara, Fazenda Boa Esperança, C. arabica cv. Mundo Novo, 16.v.2001, $3 \mathrm{f}$ e 1m; 27.ix.2002, 1f; 07.i.2003, 1f; Garça, Estação Experimental Alcides Carvalho, C. arabica cv. Mundo Novo, 07.v.2001, 1f; 07.viii.2001, 1f; 21.ix.2001, 4f; 29.i.2003, 1f; cv. Catuaí Amarelo, 06.xi.2001, 1f; cv. Icatu Vermelho, 09.v.2001, 1f; C. canephora cv. Apoatã, 29.i.2003, 1f; Franca, Sítio Ripa, C. arabica cv. Catuaí Vermelho, 20.ii.2003, 2f; Jaguariúna, Sítio São José, C. arabica, 08.xi.2003, 1f ; Tatuí, Sítio Morro Alto, C. arabica, 08.i.2004, 3f; Itirapuã, Sítio Santo Antônio, C. arabica cv. Mundo Novo, 21.i.2004, 3f; São Paulo, Instituto Biológico, C. arabica, 05.xi.2003, 5f; Cândido Mota, Distrito Frutal do Campo, C. arabica cv. Catuaí Vermelho, 10.iii.2004, 8f; Lutécia, C. arabica cv. Catuaí Vermelho, 10.iii.2004, 4f; Campinas, IAC, Coffea liberica cv. Piatã, 02.ix.2003, 6f, C. Canephora cv. Guarini, 02.ix.2003, 3f; C. canephora cv. Apoatã, 26.x.2005, 8f; Paranapuã, C. canephora cv. Apoatã, 25.iii.2004, 2f; Olímpia, Sítio São Pedro, C. arabica, 07.iv.2004, 1f; Cristais Paulista, C. arabica, 21.i.2004, 1f; Jeriquara, Fazenda Goretti, C. arabica cv. Sarchimor, 28.i.2005, 1f; Monte Alegre do Sul, Sítio São José, C. arabica cv. Mundo Novo, 30.xii.2004, 1f; 09.iii.2005, 1f; Monte Mor, Faz. Mundo Novo, C. arabica cv. Mundo Novo, 01.ix.2005, 2f; Atibaia, Sítio Kurosawa, C. arabica cv. Mundo Novo, 20.viii.2007, 1f.

Relatos prévios no Brasil: Bahia, Ceará, Maranhão, Minas Gerais, Paraíba, Pernambuco, Piauí, Rio Grande do Sul, São Paulo (Moraes \& McMurty 1983, Moraes et al. 1993, Sato et al. 1994, Gravena et al. 1994, Pallini Filho et al. 1992, Rodrigues et al. 1996, Santana \& Flechtmann 1998, Ferla \& Moraes 2002, Feres et al. 2003, Gondim Jr. \& Moraes 2001, Moraes \& McMurtry 1982, Zacarias \& Moraes 2001, Zacarias \& Moraes 2002, Mineiro \& Raga 2003, Mineiro et al. 2004, Lofego et al. 2004, Daud \& Feres 2005, Bellini et al. 2005, Spongoski et al. 2005, Demite \& Feres 2005, Furtado et al. 2005, Hernandes \& Feres 2006, Buosi et al. 2006).

9. Euseius concordis (Chant, 1959)

Typhlodromus (Amblyseius) concordis Chant, 1959: 69. Amblyseius(Iphiseius) concordis Muma, 1961:288. Amblyseius concordis Chant \& Baker, 1965: 22.

Euseius concordis Denmark \& Muma, 1973: 264, Moraes \& Oliveira, 1982: 317, Moraes \& McMurtry, 1983: 138, Moraes et al., 1986: 39, Feres \& Moraes, 1998: 127, Gondim Jr. \& Moraes, 2001: 74, Ferla \& Moraes, 2002: 1016, Moraes et al., 2004: 64 , Lofego et al., 2004: 5, Feres et al., 2005: 3, Bellini et al., 2005: 37, Furtado et al., 2005: 134, Vasconcelos et al., 2006: 93, Buosi et al., 2006: 4.

Euseius flechtmanni Denmark \& Muma, 1970: 223, 1973: 261 (Sinonímia de acordo com Moraes et al., 1982: 18)

Espécimes examinados: Jeriquara, Fazenda Boa Esperança, C. arabica cv. Mundo Novo, 09.viii.2001, 2f; 24.viii.2001, 1f; 06.x.2001, 3f; 16.ii.2002, 1f; Fazenda São Francisco, C. arabica cv. Mundo Novo, 06.iv.2002, 1f; cv. Catuaí Amarelo, 15.ii.2002, 1f; 15.v.2003, 1f; Garça, Estação Experimental Alcides Carvalho, C. arabica cv. Mundo Novo, 05.iv.2001, 4f; 23.vii.2001, 2f; 30.iv.2003, 1f; C. canephora cv. Apoatã, 09.v.2001, 7f; Assis, Pólo Regional do Médio Paranapanema, C. arabica cv. Obatã, 05.ix.2003, 14f; Gabriel Monteiro, C. arabica cv. Obatã, 05.ix.2003, 1f; Piracicaba, ESALQ/USP, C. canephora, 12.viii.2003, 3f; Jaguariúna, Sítio São José, C. arabica, 08.xi.2003, 3f; Atibaia, C. arabica cv. Catuaí Amarelo, 24.vii.2003; Olímpia, Sítio São Pedro, C. arabica, 07.iv.2004, 5f; Mococa, Pólo Regional de Mococa, C. arabica var. Tupi, 18.vi.2004, 9f; Monte Alegre do Sul, Sítio São José, C. arabica cv. Mundo Novo, 13.iv.2004, 1f; Pólo Regional do Leste Paulista, C. arabica, 01.xii.2006, 1f; Campinas, IAC, C. canephora cv. Apoatã, 26.x.2005, 1f; Monte Mor, Faz. Mundo Novo, C. arabica cv. Mundo Novo, 01.ix.2005, 1f; Maracaí, Sítio Santa Margarida, C. arabica, 31.v.2006, 11f; Atibaia, Sítio Kurosawa, C. arabica cv. Mundo Novo, 23.iv.2007, 1f.

Relatos prévios no Brasil: Ceará, Minas Gerais, Pernambuco, Piauí, Rio Grande do Sul, São Paulo (Pallini Filho et al. 1992, Moraes et al. 1993, Sato et al. 1994, Rodrigues et al. 1996, Mineiro \& Raga 2003, Mineiro et al. 2004, Lofego et al., 2004, Daud \& Feres 2005, Bellini et al. 2005, Furtado et al. 2005, Spongoski et al. 2005, Hernandes \& Feres 2006, Vasconcelos et al. 2006, Buosi et al., 2006, Ferla et al. 2007).

\section{Iphiseiodes aff metapodalis}

Espécimes examinados: Monte Alegre do Sul, Pólo Regional do Leste Paulista, C. arabica do interior de mata, 20.vi.2006, 2 f.

\section{Iphiseiodes zuluagai Denmark \& Muma, 1972}

Iphiseiodes zuluagai Denmark \& Muma, 1972: 29, Denmark \& Muma, 1973: 251, Denmark \& Muma, 1975: 287, Moraes et al., 1982: 18, Moraes et al., 1986: 61, Aponte \& McMurtry, 1995: 165, Kreiter \& Moraes, 1997: 377, Feres \& Moraes, 1998: 127, Moraes et al., 1999 (2000): 245, Zacarias \& Moraes, 2001: 581, Gondim Jr. \& Moraes, 2001: 76, Ferla \& Moraes, 2002: 1013, Moraes et al., 2004: 91, Lofego et al., 2004: 7, Feres et al., 2005: 3, Bellini et al., 2005: 37, Vasconcelos et al., 2006: 94, Buosi et al., 2006: 5 .

Amblyseius zuluagai; Moraes \& Mesa, 1988: 79, Moraes et al., 1991: 125.

Espécimes examinados: Atibaia, Sítio Kurosawa, C. arabica cv. Catuai Amarelo, 13.vi.2000, 7f, 24.vii.2003, 6f; Jeriquara, Fazenda São Francisco, C. arabica cv. Catuai Amarelo, 20.vi.2003, 9f; Piracicaba, ESALQ/USP, C. canephora, 12.viii.2003, 6f; Jaboticabal, UNESP/FCAV, C. arabica, 18.vii.2003, 14f, 1n, C. arabica, 12.viii.2003, 1f; Tietê, C. arabica cv. Obatã, 07.viii.2003, 10f; São Paulo, Instituto Biológico, 05.xi.2003, C. arabica, 1f; Monte Mor, Faz. Mundo Novo, C. arabica cv. Mundo Novo, 01.ix.2005, 1f; Campinas, CEIB, C. arabica, 27.vii.2005, 4f; Socorro, Sítio São Miguel, C. arabica, 25.v.2006, 8f.

Relatos prévios no Brasil: Bahia, Maranhão, Minas Gerais, Pernambuco, Rio Grande do Sul e São Paulo (Moraes et al. 1986, 1993, Pallini Filho et al. 1992, Sato et al. 1994, Feres \& Moraes 1998, Ferla \& Moraes 1998, 2002, Reis et al. 2000, Zacarias \& Moraes 2002, Barbosa et al. 2003, Mineiro \& Raga 2003, Mineiro et al. 2004, Lofego et al. 2004, Bellini et al. 2005, Demite \& Feres 2005, Vasconcelos et al. 2006, Buosi et al. 2006).

\section{Metaseiulus camelliae (Chant \& Yoshida-Shaul, 1983)}

Typhlodromus camelliae Chant \& Yoshida-Shaul, 1983: 1053, Feres \& Moraes 1998: 130, Zacarias \& Moraes, 2001: 583, Ferla \& Moraes, 2002: 1019.

Metaseiulus camelliae Moraes et al., 2004: 277, Lofego et al., 2004: 13 .

Espécimes examinados: Garça, Estação Experimental Alcides Carvalho, C. arabica cv. Icatu Vermelho, 03.vi.2002, 2f; Monte Alegre do Sul, Sítio São José, C. arabica cv. Mundo Novo, 02.ii.2005, 1f; 23.iii.2005, 1f.

Relatos prévios no Brasil: São Paulo, Rio Grande do Sul (Lorenzato 1988, Lorenzato et al. 1986, Sato et al. 1994, Feres \& Moraes 1998, Gondim Jr. \& Moraes 2001, Zacarias \& Moraes 
2002, Ferla \& Moraes 1998 e 2002, Mineiro et al. 2004, Lofego et al. 2004, Daud \& Feres 2005, Hernandes \& Feres 2006).

13. Neoseiulus barkeri Hughes, 1948

Neoseiulus barkeri Hughes, 1948: 141 e 1976: 343, Moraes et al., 2004: 104.

Typhlodromus barkeri, Nesbitt, 1951: 35.

Typhlodromus (Typhlodromus) barkeri, Chant, 1959: 61.

Espécime examinado: Jeriquara, Fazenda Boa Esperança,

C. arabica cv Mundo Novo, 14.ix.2002, 1f.

Relatos prévios no Brasil: Pernambuco, Piauí (Moraes et al. 1993).

\section{Neoseiulus aff. mumai}

Espécime examinado: Jeriquara, Fazenda Boa Esperança, C. arabica cv Mundo Novo, 15.v.2002, 1f.

\section{Neoseiulus transversus Denmark \& Muma, 1973}

Neoseiulus transversus Denmark \& Muma, 1973: 267, Moraes et al., 2004: 147.

Amblyseius transversus, Moraes \& McMurtry, 1983: 135.

Espécimes examinados: Jeriquara, Fazenda Boa Esperança, C. arabica cv. Mundo Novo, 15.v.2002, 1f; Garça, Estação Experimental Alcides Carvalho, C. arabica cv. Mundo Novo, 07.viii.2001, 1f, cv. Icatu Amarelo, 10.v.2001, 1f, cv. Catuai Amarelo, 05.ix.2001, 1f.

Relatos prévios no Brasil: Bahia, Pernambuco, Piauí, São Paulo e Paraíba (Denmark \& Muma 1973, Moraes \& McMurtry 1983, Ragusa \& Athias-Henriot 1983).

16. Phytoseius woodburyi De Leon, 1965

Phytoseius woodburyi De Leon, 1965: 130, Moraes et al., 2004: 257.

Espécimes examinados: Monte Alegre do Sul, Sítio São José, C. arabica cv. Mundo Novo, 05.v.2004, 1f; 26.viii.2005, 1 f.

Relatos prévios no Brasil: São Paulo (Gondim Jr. \& Moraes 2001).

17. Phytoseiulus macropilis (Banks, 1905)

Laelaps macropilis Banks, 1905: 139.

Hypoaspis macropilis (Banks), Banks, 1915: 85.

Phytoseiulus speyeri Evans, 1952: 398 (Sinonímia de acordo com Kennet, 1958: 477).

Phytoseiulus chanti Ehara, 1966: 135 (Sinonímia de acordo com Denmark \& Muma, 1973: 236).

Phytoseiulus macropilis (Banks) Cunlife \& Baker, 1953: 23, Garman, 1958: 70, Kennett, 1958: 477-78, Chant, 1959: 109, Schuter \& Pritchard, 1963: 279, Chant \& Baker, 1965: 13, Muma \& Denmark, 1970: 30, Denmark \& Muma, 1973: 236, McMurtry, 1983: 259, Denmark \& Schicha, 1983: 31, Moraes et al., 1986: 108, Kreiter \& Moraes, 1997: 378, Gondim Jr. \& Moraes, 2001: 81, Ferla \& Moraes, 2002: 1018, Moraes et al., 2004: 167, Vasconcelos et al., 2006: 94.

Espécimes examinados: Jeriquara, Fazenda São Francisco, C. arabica cv. Catuai Amarelo, 17.iv.2003, 1f; Garça, Estação Experimental Alcides Carvalho, C. arabica cv. Mundo Novo, 03.vi.2002, 3f.

Relatos prévios no Brasil: Ceará, Minas Gerais, Pernambuco, Rio Grande do Sul, São Paulo (Ehara 1966, Denmark \& Muma 1973, Rowell et al. 1978, Samways 1979, Reis et al. 2000, Mineiro \& Moraes 2001, Mineiro \& Raga 2003, Furtado et al. 2005, Vasconcelos et al. 2006, Ferla et al. 2007).
18. Proprioseiopsis cannaensis (Muma, 1962)

Amblyseiulus cannaensis Muma, 1962: 4.

Amblyseius cannaensis, Moraes \& McMurtry, 1983: 132, Moraes \& Mesa, 1988: 77, Moraes et al., 1991: 126.

Proprioseiopsis cannaensis, Muma \& Denmark, 1970: 38, Moraes \& McMurtry, 1983: 132, Kreiter \& Moraes, 1997: 379, Zacarias \& Moraes, 2001: 581, Moraes et al., 2004: 172.

Espécimes examinados: Garça, Estação Experimental Alcides Carvalho, C. canephora cv. Apoatã, 06.iv.2001, 1f; Jeriquara, Fazenda São Francisco, C. arabica cv. Catuai Amarelo, 15.v.2003, 1f.

Relatos prévios no Brasil: Bahia, Maranhão, Pernambuco, Piauí, Rio Grande do Sul e São Paulo (Moraes \& McMurtry 1983, Moraes et al. 1993, Zacarias \& Moraes 2002, Ferla et al. 2007).

19. Proprioseiopsis dominigos (El-Banhawy, 1984)

Amblyseius dominigos El-Banhawy, 1984: 130, McMurtry \& Moraes, 1989: 185, Moraes et al., 1991: 126, Feres \& Moraes, 1998:126.

Proprioseiopsis dominigos, Zacarias \& Moraes, 2001: 582, Moraes et al., 2004: 174.

Espécimes examinados: Atibaia, Sítio Kurosawa, C. arabica cv. Catuai Amarelo, 25.vii.2000, 3f; Cristais Paulista, C. arabica, 21.i.2004, 1f; Monte Alegre do Sul, Sítio São José, C. arabica cv. Mundo Novo, 07.vi.2004, 1f; 23.vi.2004, 1f; 29.vii.2004, 3f; 23.xii.2004, 2f; 23.iii.2005, 1f; Itupeva, C. arabica, 26.ii.2004, 1f.

Relatos prévios no Brasil: Espírito Santo, Minas Gerais, Santa Catarina, São Paulo (El-Banhawy 1984, Fellini \& Flechtmann 1990, Pallini Filho et al. 1992, Feres \& Moraes 1998, Mineiro \& Moraes 2001, Gondim Jr. \& Moraes 2001, Zacarias \& Moraes 2002).

20. Proprioseiopsis neotropicus (Ehara, 1966)

Amblyseius neotropicus Ehara, 1966: 133, Moraes \& Mesa, 1988: 79, Moraes et al., 1991: 127.

Proprioseiopsis neotropicus, Moraes et al., 2004: 182, Lofego et al., 2004: 9, Vasconcelos et al., 2006: 95.

Espécimes examinados: Atibaia, Sítio Jorge Kurosawa, C. arabica cv. Catuaí Amarelo, 13.vi.2000, 1 f.

Relatos prévios no Brasil: Bahia, Pernambuco, Rio Grande do Sul e São Paulo (Moraes \& Mesa 1988, Moraes et al. 1991, Ferla \& Moraes 1998 e 2002, Gondim Jr. \& Moraes 2001, Zacarias \& Moraes 2002, Lofego et al. 2004, Vasconcelos et al. 2006).

21. Proprioseiopsis ovatus (Garman, 1958)

Amblyseiopsis ovatus Garman, 1958: 78.

Amblyseius ovatus, Moraes \& McMurtry, 1983: 133, Moraes et al., 1991, 127.

Typhlodromus (Amblyseius) ovatus Chant, 1959: 90.

Proprioseiopsis ovatus, Denmark \& Muma, 1973: 237, Moraes et al., 2004: 183, Buosi et al., 2006: 6.

Espécimes examinados: Garça, Estação Experimental Alcides Carvalho, C. arabica cv. Icatu Amarelo, 06.xi.2001, 1f; Jeriquara, Fazenda São Francisco, C. arabica cv. Catuai Amarelo, 18.xii.2002, 1f.

Relatos prévios no Brasil: São Paulo (Denmark \& Muma 1973, Gondim Jr. \& Moraes 2001, Buosi et al. 2006).

22. Typhlodromus transvaalensis (Nesbit, 1951)

Kampimodromus transvaalensis Nesbitt, 1951: 55.

Typhlodromus jackmickleyi De Leon, 1958: 75.

Typhlodromus pectinatus Athias-Henriot, 1958, 179. 
Neoseiulus transvaalensis (Nesbitt), Muma, 1961: 295.

Clavidromus jackmickleyi (De Leon) Muma, 1961: 296.

Clavidromus transvaalensis Muma \& Denmark, 1968: 238, Muma \& Denmark, 1970: 128, Denmark \& Muma, 1973: 272.

Typhlodromus transvaalensis, Chant, 1955: 498, Ferla \& Moraes, 2002: 1020, Moraes et al., 2004: 353, Vasconcelos et al., 2006: 95, Ferla et al., 2007: 3.

Espécimes examinados: Garça, Estação Experimental Alcides Carvalho, C. arabica cv. Mundo Novo, 06.xi.2001, 1f; 15.ii.2002, 1f e 04.iii.2002, 1f; $C$. arabica cv. Icatu Amarelo, 01.iv.2002, 1f; $C$. arabica cv. Catuaí Amarelo, 06.ix.2002, 1f e 04.xii.2002, 1f e 1n; Jeriquara, Fazenda Boa Esperança, C. arabica cv. Mundo Novo, 02.iii.2002, 1f, 12.iv.2002, 5f e 21.v.2003, 1f; Fazenda São Francisco, C. arabica cv. Mundo Novo, 15.ii.2002, 1f e 06.iv.2002, 2f; Franca, Fazenda da Mata, C. arabica cv. Catuaí Vermelho, 20.ii.2003, 1f; Lutécia, C. arabica cv. Catuaí Vermelho, 10.iii.2004, 4f; Monte Alegre do Sul, Sítio São José, C. arabica cv. Mundo Novo, 23.xii.2004, 1f; Cristais Paulista, C. arabica, 21.i.2004, 1f; Monte Alegre do Sul, Sítio São José, C. arabica cv. Mundo Novo, 22.xii.2006, 2f.

Relatos prévios no Brasil: Pernambuco, São Paulo, Rio Grande do Sul (Denmark \& Muma 1973, Ferla \& Moraes 1998 e 2002, Gondim Jr. \& Moraes 2001, Mineiro et al. 2004, Vasconcelos et al. 2006, Ferla et al. 2007)

\section{Incertae Sedis}

\section{Africoseius sp.}

Espécimes examinados: Atibaia, Sítio Kurosawa, C. arabica cv. Catuaí Amarelo, 27.vi.2000, 2f e 11.vii.2000, 1f e 1dn; C. arabica cv. Mundo Novo, 23.viii.2007, if e 22.ii.2007, 1f; Jeriquara, Fazenda Boa Esperança, C. arabica cv. Mundo Novo, 12.iv.2002, 1f; 16.ii.2003, 1f; 28.ii.2003, 1f; 29.iii.2003, 1f e 26.iv.2003, 2f; Garça, Estação Experimental Alcides Carvalho, C. arabica cv. Mundo Novo, 01.iv.2002, 1f e 03.vii.2002, 1f; Franca, Fazenda da Mata, C. arabica cv. Catuaí Vermelho, 1n, 20.ii.2003; Monte Alegre do Sul, Sítio São José, C. arabica cv. Mundo Novo, 14.i.2005, 1 pn; 02.ii.2005, 1f e 16.iii.2005, 1 dn; Pólo regional do Leste Paulista, C. arabica sob mata, 01.xii.2006, 1dn.

\section{Discussão}

Pelo menos quatro das 39 espécies registradas podem ser determinadas como novas, devendo ser descritas oportunamente. Dentre as seis famílias de ácaros encontradas, aquelas que abrigaram as maiores riquezas de espécies foram Phytoseiidae (22) e Ascidae (10). Várias espécies de ácaros da família Ascidae são predadores de vida livre que vivem no solo, plantas e produtos armazenados (Gerson et al. 2003). Entre os Phytoseiidae, a mais importante família de ácaros predadores em plantas, as espécies mais comuns foram E. citrifolius. E. concordis e I. zuluagai, encontrados na maioria dos locais amostrados.

Este é o primeiro inventário de acarofauna realizado em diferentes regiões do Estado de São Paulo, com amostragem de todos os grupos taxonômicos de ácaros plantícolas da ordem Mesostigmata.

\section{Agradecimentos}

À FAPESP (Fundação de Amparo à Pesquisa do Estado de São Paulo) pela bolsa de pós-doutorado ao primeiro autor (Processo \# 2006/05798-7).

\section{Referências Bibliográficas}

APONTE, O. \& McMURTRY, J.A. 1995. Revision of the genus Iphiseiodes De Leon (Acari: Phytoseiidae). Intern. J. Acarol. 21(3):165-183.

ATHIAS-HENRIOT, C. 1957. Phytoseiidae et Aceosejidae (Acarina: Gamasina) d'Algérie, I Genres Blattisocius Keegan, Iphiseius Berlese, Amblyseius Berlese, Phytoseius Ribaga, Phytoseiulus Evans. Bull. Soc. Hist. Nat. Afr. Nord. 48:319-352.

ATHIAS-HENRIOT, C. 1958. Contribution à la Connaissance du genre Typhlodromus Scheuten. (Acariens Parasitiformes, Phytoseiidae): description de deux espècies nouvelles d'Algérie et clé des esècies du groupe filandicus. Rev. Pathol. Végét. d'Entomol. Agric. 37(2):179-186.

ATHIAS-HENRIOT, C. 1959. Phytoseiidae et Aceosejidae (Acarina, Gamasina) d'Algérie. III. Contribution aux Aceosejinae. Bull. Soc. Hist. Nat. 50:158-195

BAKER, E.W. \& WHARTON, G.W. 1952. An introduction to acarology. Macmillan, New York. 465p.

BANKS, N. 1905. Descriptions of some new mites. Proc. Ent. Soc. Wash. 7:133-142.

BANKS, N. 1915. The Acarina or mites: a review of the group for the use of economic entomologists. U.S. Dept. Agr. Rep. 108:1-153.

BARBOSA, D.G.F., GONDIM Jr., M.G.C., BARROS, R. \& OLIVEIRA, J.V. 2003. Diversidade de ácaros em aceroleira (Malpighia emarginata A.DC.) na Universidade Federal de Pernambuco em Recife, PE. Neotrop. Entomol. 32(4):577-583.

BELLINI, M.R., MORAES, G.J. \& FERES, R.J.F. 2005. Plantas de ocorrência espontânea como substratos alternativos para fitoseídeos (Acari, Phytoseiidae) em cultivos de seringueira Hevea brasiliensis Muell. Arg. (Euphorbiaceae). Rev. Bras. Zool. 22(1):35-42.

BERLESE, A. 1892. Acari, myriopoda et Scorpiones hucusque in Italia reperta. Ordo Mesostigmata (Gamasidae). Tipografia del Seminario, Padova. vol. 11, 143p.

BERLESE, A. 1913. Acarotheca itálica: Fasciculi Ius et Ilus. Ricci, Firenze. $221 \mathrm{p}$.

BERLESE, A. 1918. Centuria quarta di Acari nuovi. Redia, 12(2):115-192.

BRAM, R.A. 1956. A new predatory mite from insect culture. Proc. Ent. Soc. Wash. 58:292-294.

BUOSI, R., FERES, R.J.F., OLIVEIRA,A.R., LOFEGO,A.C. \& HERNANDES F.A. 2006. Ácaros plantícolas (Acari) da "Estação Ecológica de Paulo de Faria", Estado de São Paulo, Brasil. Biota Neotrop. 6(1): http://www. biotaneotropica.org.br/v6n1/pt/abstract?article+bn02006012006. (último acesso em 18/09/2007)

BURKHARDT, F. 1920. Zur biologie der mehlmotte (Phestia Kuehniella Zeller). Zeitsch. Angewan. Entomol. 6(1):25-60.

CHAMP, B.R. 1965. An investigation of peanut storage pests in Queensland. 1. Introduction, species and pest status. Queensland J. Agric. Anim. Sci. 22:227-240.

CHAMP, B.R. 1966. Insects and mites associated with stored products in Queensland. 4. Acarina and Pseudoscorpiones. Queensland J. Agric. Anim. Sci. 23:197-210.

CHANT, D.A. \& BAKER, E.W. 1965. The Phytoseiidae (Acarina) of Central America. Mem. Entomol. Soc. Can. 41:1-56.

CHANT, D.A. \& McMURTRY, J.A. 1994. A review of the subfamilies Phytoseiinae and Typhlodrominae (Acari: Phytoseiidae). Internat. J. Acarol. 20(4):222-311.

CHANT, D.A. \& YOSHIDA-SHAUL, E. 1983 A world review of five similar species groups in the genus Typhlodromus Scheuten: Part II. The conspicuus and cornus groups (Acarina: Phytoseiidae). Can. J. Zool. 61:1041-1057.

CHANT, D.A. 1955. Notes on mites of the genus Typhlodromus Scheuten, 1857 (Acarina: Laelapidae), with descriptions of the males of some species and the female of a new species. Canad. Entomol. 87(11):296-503.

CHANT, D.A. 1959. Phytoseiid mites (Acarina: Phytoseiidae). Part I. Bionomics of seven species in southeastern England. Part II. A taxonomic 
review of the family Phytoseiidae, with descriptions of 38 new species. Can. Entomol. 91(12):1-166.

CHANT, D.A. 1963. The subfamily Blattisocinae Garman (=Aceosejinae Evans) (Acarina: Blattisocidae Garman) (=Aceosejidae Baker and Warton) in North America, with descriptions of new species. Can. J. Zool. 41:243-305.

CHAUDHRI, W.M. 1968. Six new species of mites of the genus Amblyseius (Phytoseiidae) from Pakistan. Acarologia 10(4):550-562.

CUNLIFE, F. \& BAKER, E.W. 1953. A guide to the predatory phytoseiid mites of the United States. Pinellas Biol. Lab. Pub. Inc. USA, 28p.

DANESHVAR, H. \& DENMARK, H.A. 1982. Phytoseiids of Iran (Acarina: Phytoseiidae). Intern. J. Acarol. 8(1):3-14.

DAUD, R.D. \& FERES, R.J.F. 2005. Diversidade e flutuação populacional de ácaros (Acari) em Maeba fistulifera Mart. (Euphorbiaceae) de dois fragmentos de mata estacional semidecídua em São José do Rio Preto, SP. Neotrop. Entomol. 34(2):191-201, 2005.

De LEON, D. 1958. Four new Typhlodromus from southern Florida. Fla. Entomol. 41(2):73-76.

De LEON, D. 1961. Eight new Amblyseius from México (Acarina: Phytoseiidae). Fla. Entomol. 44(2):85-91.

De LEON, D. 1962. The cervices of some phytoseiid type specimens (Acarina: Phytoseiidae). Acarologia 4(2):174-176.

De LEON, D. 1965. Phytoseiidae mites from Puerto Rico with descriptions of new species (Acarina: Mesostigmata). Fla. Entomol. 48(2):121-131.

De LEON, D. 1966. Phytoseiidae of Bristish Guyana with keys to species (Acarina: Mesostigmata). In Studies the fauna of Suriname and others Guyanas, 8: 81-102.

De LEON, D. 1967. Some mites of the Caribbean Área. Part I. Acarina on plants in Trinidad, West Indies. Allen Press Inc., Lawrence. Kansas, p. 1-66.

DEMITE, P.R. \& FERES, RJF. 2005. Influência de vegetação vizinha na distribuição de ácaros em seringal (Hevea brasiliensis Muell. Arg., Euphorbiaceae) em São José do rio Preto, SP. Neotrop. Entomol. 34(5):829-836.

DENMARK, H.A. \& MUMA, M.H. 1970. Some phytoseiid mites of Paraguay (Phytoseiidae: Acarina). Fla. Entomol. 53(4):219-227.

DENMARK, H.A. \& MUMA, M.H. 1972. Some phytoseiids of Colombia (Acarina: Phytoseiidae). Fla. Entomol. 55(1):19-29.

DENMARK, H.A. \& MUMA, M.H. 1973. Phytoseiid mites of Brazil (Acarina: Phytoseiidae). Rev. Bras. Biol. 33(2):235-276.

DENMARK, H.A. \& MUMA, M.H. 1975. The Phytoseiidae (Acari: Mesostigmata) of Puerto Rico. J. Agric. Univ. 59(4):279-304.

DENMARK, H.A. \& MUMA, M.H. 1989. A revision of the genus Amblyseius Berlese, 1914 (Acari: Phytoseiidae). Occas. Pap. Flo. Sta. Coll. Arthr. 4:1-149.

DENMARK, H.A. \& SCHICHA, E. 1983. Revision of the genus Phytoseiulus Evans (Acarina: Phytoseiidae). Intern. J. Acarol. 9(1):27-35.

DOMROW, R. 1979. Ascid and amerosiid mites phoretic on Australian mammals and birds. Rec. West. Austr. Mus. 8:(1):97-116.

EHARA, S. 1966. Some mites associated with plants in the State of São Paulo, Brazil, with a list of plant mites of South America. Jap. J. Zool. 15(2):129-150.

El-BANHAWY, E.M. 1984. Description of some phytoseiid mites from Brazil (Acarina: Phytoseiidae). Acarologia, 25(2):125-144.

EVANS, G.O. 1952. On a new predatory mite of economic importance. Bull. Ent. Res. 43:397-401

EVANS, G.O. 1958. A revision of the British Aceosejinae (Acarina: Mesostigmata). Proc. Zool. Soc. London, 131:177-229.

FENILLI, R. \& FLECHTMANN, C.H.W. 1990. Ácaros do pinheiro-do-Paraná em Lages, Santa Catarina. An. Esc. Sup. 47(1):243-250.

FERES, R.J.F. \& MORAES, G.J. 1998. Phytoseiidae mites (Acari: Phytoseiidae) from wood areas in the State of São Paulo, Brazil. Syst. App. Acarol. 3:125-32.
FERES, R.J.F., BELLINI, M.R. \& ROSSA-FERES, D.C. 2003. Ocorrência e diversidade de ácaros (Acari, Arachnida) associados à Tabebuia róseo-alba (Ridl.) Sand (Bignoniaceae), no município de São José do Rio Preto, São Paulo, Brasil. Rev. Bras. Zool. 20(3):373-378.

FERES, R.J.F., LOFEGO, A.C. \& OLIVEIRA, A.R. 2005. Ácaros plantícolas (Acari) da "Estação Ecológica do Noroeste Paulista", Estado de São Paulo, Brasil. Biota Neotrop. 5(1): http://www.biotaneotropica.org.br/v5n1/pt/ abstract?article+bn00405012005. (último acesso em 18/09/2007)

FERLA, N.J. \& MORAES, G.J. 1998. Ácaros predadores em pomares de maçã no Rio Grande do Sul. An. Soc. Entomol. Brasil, 27(4):649-654.

FERLA, N.J. \& MORAES, G.J. 2002. Ácaros predadores (Acari) em plantas nativas e cultivadas do Estado do Rio Grande do Sul, Brasil. Rev. Bras. Zool. 19(4):1011-1031.

FERLA, N.J., MARCHETTI, M.M. \& GONÇALVES, D. 2007. Ácaros predadores (Acari) associados à cultura do morango (Fragaria $\mathrm{sp,}$ Rosaceae) e plantas próximas no Estado do Rio Grande do Sul. Biota Neotrop. 7(2): http://www.biotaneotropica.org.br/v7n2/pt/ abstract?article+bn01807022007. (último acesso em 18/09/2007)

FLEChTMANN, C.H.W. 1967a. Phytoseiidae do Estado de São Paulo (Acarina: Mesostigmata). An. Esc. Sup. Agric. Luiz de Queiroz, $24: 247-248$

FLECHTMANN, C.H.W. 1967b. Os ácaros do cafeeiro. An. Esc. Sup. Agric. Luiz de Queiroz, 24:91-95. FLECHTMANN, C.H.W. 1968. Ácaros do cacaueiro, cafeeiro e do chá. Universidade de São Paulo, Piracicaba. Boletim de Divulgação n ${ }^{\circ} 6$.

FLECHTMANN, C.H.W. 1976. Observações sobre dois ácaros (Mesostigmata, Acari) de vida livre. An. Soc. Entomol. Brasil, 5(1):95-96.

FLECHTMANN, C.H.W. 1981. New records of mites from Brazil with description of two new species in the genus Oligonychus Berlese (Acari, Tetranychidae). Rev. Bras. Biol. 41(4):861-866.

FLECHTMANN, C.H.W. 1986. Ácaros em produtos armazenados e na poeira domiciliar. Fundação de Estudos Agrários Luiz de Queiroz - FEALQ, Piracicaba.

FURTADO, I.P., KREITER, S., MORAES, G.J., TIXIER, M.S., FLECHTMANN, C.H.W. \& KNAPP, M. 2005. Plant mites (Acari) from Northeasthern Brazil, with descriptions of two new species of the family Phytoseiidae (Mesostigmata). Acarologia, 14(2-3):131-143.

GARMAN, P. 1948. Mite species from apple trees in Connecticut. Connecticut Agric. Exper. Sta. Bull. 520:1-27.

GARMAN, P. 1958. New species belonging to the genera Amblyseius and Amblyseiopsis with keys to Amblyseius, Amblyseiopsis and Phytoseieulus. An. Entomol. Soc. Am. 51(1):69-79.

GERSON, U., SMILEY, R.L. \& OCHOA, R. 2003. Mites (Acari) for pest control. Blackwell Science Ltd., UK.

GONDIM Jr., M.G.C. \& MORAES, G.J. 2001. Phytoseiid mites (Acari: Phytoseiidae) associated with palm trees (Arecaceae) in Brazil. Syst. App. Acarol. 6:65-94.

GRAVENA, S., BENETOLI, I., MOREIRA, P.H.R. \& YAMAMOTO, P.T. 1994. Euseius citrifolius Denmark \& Muma predation on citrus leprosis mite Brevipalpus phoenicis (Geijskes) (Acari: Phytoseiidae: Tenuipalpidae). An. Soc. Entomol. Brasil, 23(2):209-218.

HAINES, C.P. 1978. A revision of the genus Blattisocius Keegan (Mesostigmata: Ascidae) with especial reference to B. tarsalis (Berlese) and the description of a new species. Acarologia, 20(1):19-38.

HALLIDAY, R.B., WALTER, D.E. \& LINDQUIST, E.E. 1998. Revision of the Australian Ascidae (Acarina: Mesostigmata). Invert. Taxon. 12:1-54.

HERNANDES, F.A. \& FERES, R.J.F. 2006. Diversidade e sazonalidade de ácaros (Acari) em seringal (Hevea brasiliensis, Muell. Arg.) no noroeste do Estado de São Paulo. Neotrop. Entomol. 35(4):523-535.

HUGHES, A.M. 1948. The mites associated with stored food products. Ministry of Agriculture; Fisheries and Food, London.

HUGHES, A.M. 1961. The mites of stored food. Ministry of Agriculture; Fisheries and Food, London. Technical Bulletin $n^{\circ} 9$.

HUGHES, A.M. 1976. The mites of stored food and houses. 2 ed. Ministry of Agriculture, London, p. 1-400. Technical Bulletin $n^{\circ} 9$ 
KARG, W. 1985. Die Raubmilbengattung Proctolaelaps Berlese, 1923. Zool. Jahr. Abt. Syst. Ökol. 112:185-206.

KARG, W. 1988. Zur Kenntnis der Gattung Proctolaelaps Berlese, 1923 (Acarina, Parasitiformes). Zool. Jahr. Abt. Syst. Ökol. 115:441-454.

KARG, W. 1993. Acari (Acarina), Milben. Parasitiformes (Anactinochaeta). Cohors Gamasina Leach. Raubmilben. 2ed. Die Tierwelt, Deutschlands, v. 59 , p. $1-523$.

KEEGAN, H.L. 1944. On a new genus and species of parasitid mite. J. Parasit. 30:181-183.

KENNETT, C. 1958. Some predaceous mites mites of the subfamilies Phytoseiinae and Aceosejinae (Acarina: Phytoseiidae, Aceosejidae) from Central California with descriptions of new species. An. Entomol. Soc. Am. 51(5):471-479.

KREITER, S. \& MORAES, G.J. 1997. Phytoseiid mites (Acari: Phytoseiidae) from Guadaloupe and Martinique. Fla. Entomol. 80(3):376-383.

LINDQUIST, E.E. \& CHANT, D.A. 1964. A redescription of Aceodromus Muma and its transfer to the Blattisocinae (Acarina: Blattisociidae). Can. Entomol. 96:500-507.

LINDQUIST, E.E. \& EVANS, G.O. 1965. Taxonomic concepts in the Ascidae, with a modified setal nomenclature for the idiosoma of the Gamasina (Acarina: Mesostigmata). Mem. Entomol. Soc. Can. 47:1-64.

LINDQUIST, E.E. \& HUNTER, P.E. 1965. Some mites of the genus Proctolaelaps Berlese (Acarina: Blattisocidae) associated with forest insect pests. Can. Entomol. 97:16-32.

LOFEGO, A.C., MORAES, G.J. \& CASTRO, L.A.S. 2004. Phytoseiid mites (Acari: Phytoseiidae) on Myrtaceae in the State of São Paulo, Brazil. Zootaxa, 516:1-18.

LORENZATO, D. 1987. Controle biológico de ácaros fitófagos na cultura da macieira no município de Farroupilha, RS. Agron. Sulriogr. 23(2):167-183.

LORENZATO, D. 1988. Ocorrência e controle biológico de ácaros fitófagos em fruteiras rosáceas. Ipagro Inf. 31:93-96.

LOREnZATO, D., GREllman, E.O., Chouene, E.C. \& NEYER-CACHAPUZ, L.M. 1986. Flutuação populacional de ácaros fitófagos e seus predadores associados a cultura da macieira (Malus domestica Bork) e efeitos do controles químicos e biológicos. Agron. Sulriogr. 22(2):215-242.

McGRAW, J.R. \& FARRIER, M.H. 1969. Mites of the superfamily Parasitoidea (Acarina: Mesostigmata) associated with Dendroctonus and Ips (Coleoptera: Scolytidae). Agric. Exper. Stat. Tec., North Carolina. Bulletin no. 192

McMURTRY, J.A. \& MORAES, G.J. 1984. Some phytoseiid mites from the South Pacific, with descriptions of new species and definition of the Amblyseius largoensis species group. Intern. J. Acarol. 10(1):27-37.

McMURTRY, J.A. \& MORAES, G.J. 1989. Some phytoseiid mites from Peru with descriptions of four new species (Acari: Phytoseiidae). Intern. J. Acarol. 15(3):179-188.

McMURTRY, J.A. 1983. Phytoseiidae mites from Guatemala, with descriptions of two new species and redefinitions of the genera Euseius, Typhloseiopsis, and the Typhlodromus occidentalis species group (Acari: Mesostigmata). Intern. J. Entomol. 25(4):249-272.

MINEIRO, J.L.C. \& MORAES, G.J. 2001. Gamasida (Arachnida: Acari) edáficos de Piracicaba, Estado de São Paulo. Neotrop. Entomol. 30(3):379-385.

MINEIRO, J.L.C. \& RAGA, A. 2003. Ocorrência de ácaros (Arachnida: Acari) em plantas de lichia (Litchi chinensis Sonn) no Estado de São Paulo. Arq. Inst. Biol. 70(3):1-4.

MINEIRO, J.L.C., LOFEGO, A.C., RAGA, A. \& MORAES, G.J. 2005. Primeiros registros dos ácaros Amblyseiella setosa Muma (Phytoseiidae) e Tuckerella pavoniformis (Ewing) (Tuckerellidae) no Brasil. Arq. Inst. Biol. 72(3):395-396.

MINEIRO, J.L.C., RAGA, A. \& LOFEGO, A.C. 2004. Ocorrência de ácaros (Arachnida: Acari) em aceroleira (Malpighia emarginata A.DC.) no Estado de São Paulo. Arq. Inst. Biol. 71(supl.):1-4.
MINEIRO, J.L.C., SATO, M.E., RAGA, A., ARTHUR, V., MORAES, G.J., SARRETA, F.O. \& CARRIJO, A. 2006. Diversidade de ácaros (Arachnida: Acari) em Coffea arabica L. cv. Mundo Novo, nos municípios de Jeriquara e Garça, Estado de São Paulo. Biota Neotrop. 6(2): http:// www.biotaneotropica.org.br/v6n2/pt/abstracts?article+bn01106022006. (último acesso em 03/09/2006)

MINEIRO, J.L.C., SATO, M.E., RAGA, A., SOUZA FILHO, M.F., SILOTO, R.C., MORAES, G.J. \& SPONGOSKI, S. 2001. Distribuição da acarofauna em cafeeiro (Coffea arabica var. Catuaí Amarelo) em Atibaia, SP. In II Simpósio de Pesquisa dos cafés do Brasil. Vitória, Espírito Santo, p.1471-1474.

MORAES, G.J. \& FLECHTMANN, C.W.H. 2008. Manual de acarologia: acarologia básica e ácaros de plantas cultivadas no Brasil. Editora Holos, Ribeirão Preto.

MORAES, G.J. \& McMURTRY, J.A. 1982. Biology of Amblyseius citrifolius (Denmark \& Muma). Hilgardia, 49(1):1-29.

MORAES, G.J. \& McMURTRY, J.A. 1983. Phytoseiid mites (Acarina) of Northeastern Brazil with descriptions of four new species. Intern. J. Acarol. 9(3):131-148.

MORAES, G.J. \& MESA, N.C. 1988. Mites of the family Phytoseiidae (Acari) in Colombia, with descriptions of three new species. Intern. J. Acarol. 14(2):71-88.

MORAES, G.J. \& OLIVEIRA, J.V. 1982. Phytoseiid mites of coastal Pernambuco, in northeastern Brazil. Acarologia 23(4):315-318.

MORAES, G.J., ALENCAR, J.A., LIMA, J.L.S., YANINEK, J.S. \& DELALIBERA Jr., I. 1993. Alternative plant habitats for common phytoseiid predators of the cassava green mite (Acari: Phytoseiidae, Tetranychidae) in northeast Brazil. Exp. App. Acarol. 17(1-2):77-90.

MORAES, G.J., DENMARK, H.A. \& GUERRERO, J.M. 1982. Phytoseiid mites of Colombia (Acarina: Phytoseiidae). Intern. J. Acarol. 8(1):15-22

MORAES, G.J., KREITER, S. \& LOFEGO, A.C. 1999. Plant mites (Acari) of the French Antilles. 3. Phytoseiidae (Gamasida). Acarologia, 40(3):237-264

MORAES, G.J., McMURTRY, J.A. \& DENMARK, H.A. 1986. A catalog of the mite family Phytoseiidae: references to taxonomy, synonymy, distribution and habitat. Brasília, EMBRAPA; DDT. 353p.

MORAES, G.J., McMURTRY, J.A., DENMARK, H.A. \& CAMPOS, C.B. 2004. A revised catalog of the mite family Phytoseiidae. Zootaxa, 434:1-494.

MORAES, G.J., MESA, N.C. \& BRAUN, A. 1991. Some phytoseiid mites of Latin America (Acari: Phytoseiidae). Intern. J. Acarol. 17(2):117-139.

MUMA, M.H. \& DENMARK, H.A. 1968. Some generic descriptions and name changes in the family Phytoseiidae (Acarina: Mesostigmata). Fla. Entomol. 51(4):229-240.

MUMA, M.H. 1955. Phytoseiidae (Acarina) associated with citrus in Florida. An. Entomol. Soc. Am. 48(4):262-272.

MUMA, M.H. 1961. Subfamilies, genera and species of Phytoseiidae (Acarina: Mesostigmata). Bull. Fla. State Mus. 5(7):267-302.

MUMA, M.H. 1962. New Phytoseiidae (Acarina: Mesostigmata) from Florida. Fla. Entomol. 45(1):1-10.

MUMA, M.H., DENMARK, H.A. \& De LEON, D. 1970. Phytoseiidae of the Florida: arthropods of Florida and neighboring land areas. Florida Department of Agriculture and Consumer Services; Division of Plant Industry, Gainnesville.

NESBITT, H.H.J. 1951. A taxonomic study of the Phytoseiinae (Family Laelaptidae) predaceous upon Tetranychidae of economic importance. Zool. Verh. (12):1-64.

OUDEMANS, A.C. 1901. Notes on Acari. Third series. Tijdschrift voor Entomologie. Dierk. Vereen. 7:50-88.

OUDEMANS, A.C. 1929. Acarologische aanteekeningen. C. Entomol. Ber. 8(170):28-36.

PALLINI FILHO, A., MORAES, G.J. \& BUENO, V.H.P. 1992. Ácaros associados ao cafeeiro (Coffea arabica L.) no sul de Minas Gerais. Ciênc. Prát. 16(3):303-307. 
RAGUSA, S. \& ATHIAS-HENRIOT, C. 1983. Observations on the genus Neoseiulus Hughes (Parasitiformes, Phytoseiidae). Redefinition. Composition. Geography. Description of two new species. Rev. Suis. Zool. 90(3):657-678.

REIS, P.R. \& PASCHOAL, A.D. 1968. Alguns ácaros de produtos armazenados do Estado de São Paulo. 60(2):73-74.

REIS, P.R., CHIAVEGATO, L.G., ALVES, E.B. \& SOUZA, E.O. 2000. Ácaros da família Phytoseiidae associados aos citros no município de Lavras, Sul de Minas Gerais. An. Soc. Entomol. Brasil 29(1):95-104.

RODRIGUES, G.S., PARAÍBA, L.C. \& MORAES, G.J. 1996. Pairwise association as a criterion for the selection of collection sites of natural enemies of the cassava green mite, Mononychellus tanajoa (Bondar). Scien. Agric. 53(2-3):324-331.

ROWELL, H.J., CHANT, D.A. \& HANSELL, R.I.C. 1978. The determination of setal homologies and setal patterns on the dorsal shield in the family Phytoseiidae (Acarina: Mesostigmata). Can. Entomol. 110:859-876.

SAMWAYS, M.J. 1979. Immigration, population growth and mortality of insects and mites on cassava in Brazil. Bull. Entomol. Res. 68:491-505.

SANTANA, D.L.Q. \& FLECHTMANN, C.H.W. 1998. Mite (Arthropoda: Acari) associates of palms (Arecaceae) in Brazil. Rev. Bras. Zool. 15(4):959-963.

SATO, M.E., RAGA, A., CERÁVOLO, L.C., ROSSI, A.C. \& POTENZA, M.R. 1994. Ácaros predadores em pomar cítrico de Presidente Prudente, estado de São Paulo. An. Soc. Entomol. Brasil 23(3):435-441.

SCHUTER, R.O. \& PRITCHARD, A.E. 1963. Phytoseiid mites of California. Hilgardia 34(7):191-285.

SCHWEIZER, J. 1949. Die Landmilben des Schweizerischen Nationalparkes. Erg. Wissens. Unters. Scheiz. Nat. 2:1-99.

SCHWEIZER, J. 1961. Die Landmilben der Schweiz (Mittelland, Jura und Alpen): parasitiformes Reuter. Denksc. Schweiz. Naturf. Gesells. 84.

SOUZA, J.M., GONDIM Jr., M.G.C., BARROS, R. \& OLIVEIRA, J.V. 2005. Ácaros em produtos armazenados comercializados em supermercados e feiras livres da cidade de Recife. Neotrop. Entomol. 34(2):303-309.

SPONGOSKI, S., REIS, P.R. \& ZACARIAS, M.S. 2005. Acarofauna da cafeicultura de cerrado em Patrocínio, Minas Gerais. Ciên. Agrotéc. 29(1):9-17.
TREAT, A.E. 1975. Mites of moths and butterflies. Comstock Publishing Associates, Ithaca; New York.

VASCONCELOS, G.J.N., SILVA, F.R., BARBOSA, D.G.F., GONDIM JR., M.G.C. \& MORAES, G.J. 2006. Diversidade de fitoseídeos (Acari: Phytoseiidae) em fruteiras tropicais no Estado de Pernambuco, Brasil. Magistra, 18(2):90-101.

VITZTHUM, H.G. 1930. Acarologische Beobachtungen. Zool. Jahr. (Syst.) Bd. 59(1):281-350.

VOIGTS, H. \& OUDEMANS, A.C. 1905. Zur Kenntnis der Milben-Fauna von Bremen. Abhandl. Herausg. Naturwis. Ver. Bremen 18:199-252.

WALTER, D.E., HALLIDAY, R.B. \& LINDQUIST, E.E. 1993. A review of the genus Asca (Acarina: Ascidae) in Australia, with descriptions of three new leaf-inhabiting species. Invert. Taxon. 7:1327-1347.

WESTERBOER, I. \& BERNHARD, F. 1963. Die familie Phytoseiidae Berlese 1916. In Beiträge zur Systematik und Ökologie Mitteleuropäischer Acarina. Mesostigmata 1. (H.J. Stammer, ed.). Akademische Verlagsgesellschaft Geest \& Portig K.-G., Leipzig, p. 451-777.

WESTERBOER, I. 1963. Die familie Podocinidae Berlese, 1916. In Beiträge zur Systematik und Ökologie Mitteleuropäischer Acarina. Mesostigmata 1. (H.J. Stammer, ed.). Akademische Verlagsgesellschaft Geest \& Portig K.-G., Leipzig, p. 179-450.

WOMERSLEY, H. 1954. Species of the subfamily Phytoseiinae (Acarina: Laelaptidae) from Australia. Austr. J. Zool. 2:169-191.

ZACARIAS, M.S. \& MORAES, G.J. 2001. Phytoseiid mites (Acari) associated with rubber trees and other euphorbiaceous plants in southeastern Brazil. Neotrop. Entomol. 30(4):579-586.

ZACARIAS, M.S. \& MORAES, G.J. 2002. Mite diversity (Arthropoda: Acari) on euphorbiaceous plants in three localities in the State of São Paulo. Biota Neotrop. 2(2): http://www.biotaneotropica.org.br/v2n2/pt/ abstract?article+BN00802022002. (ultimo acesso em 18/01/2005)

ZACHER, F. 1927. Die vorrats - Speicher - und Materialschadlinge und ihre Bekampfung. Paul Parey, Berlin.

Recebido em 29/01/08

Versão reformulada recebida em 10/12/08

Publicado em 12/01/09 